\title{
A comprehensive study of layer-specific morphological changes in the microstructure of carotid arteries under uniaxial load
}

\author{
Witold Krasny ${ }^{1,2,3,4}$. Claire Morin ${ }^{1,2,3}$. \\ Hélène Magoariec $^{4}$. Stéphane Avril ${ }^{1,2,3 *}$
}

Received: date / Accepted: date

\begin{abstract}
The load bearing properties of large blood vessels are principally conferred by collagen and elastin networks and their microstructural organization plays an important role in the outcomes of various arterial pathologies. In particular, these fibrous networks are able to rearrange and reorient spatially during mechanical deformations. In this study, we investigate for the first time whether these well-known morphological rearrangements are the same across the whole thickness of blood vessels, and subsequently if the underlying mechanisms that govern these rearrangements can be predicted using affine kinematics. To this aim, we submitted rabbit carotid samples to uniaxial load in three distinct deformation directions, while recording live images of the 3D microstructure using multiphoton microscopy. Our results show that the observed realignment of collagen and elastin in the media layer, along with elastin of the adventitia layer, remained limited to small angles that can be predicted by affine kinematics. We show also that collagen bundles of fibers in the adventitia layer behaved in significantly different fashion. They showed a remarkable capacity to realign in the direction of the load,

1. Ecole Nationale Suprieure des Mines de Saint-Etienne, CIS-EMSE, SAINBIOSE, F-42023 Saint Etienne, France

2. INSERM, U1059, F-42000 Saint Etienne, France

3. Université de Lyon, SAINBIOSE, F-42000 Saint Etienne, France

4. Laboratoire de Tribologie et Dynamique des Systèmes, CNRS UMR 5513, Université de Lyon, Ecole Centrale Lyon, France

*correspondance to: avril@emse.fr
\end{abstract}


whatever the loading direction. Measured reorientation angles of the fibers were significantly higher than affine predictions. This remarkable property of collagen bundles in the adventitia was never observed before, it shows that the medium surrounding collagen in the adventitia undergoes complex deformations challenging traditional hyperelastic models based on mixture theories.

Keywords Carotid artery · microstructure · adventitia $\cdot$ media $\cdot$ uniaxial load

\section{Introduction}

2 Cardiovascular disorders are a significant public health issue affecting ageing populations globally and causing considerable public health expenses (31\% of total mortality in 2012 - source: World Health Organization). In numerous cases, the disorder involves significant changes in the vascular mechanical properties, generating extensive studies about arterial biomechanics and mechanobiology. In this respect, a common approach to vascular biomechanics consists in submitting samples of arterial tissue to mechanical bench tests in order to characterize their macroscopic mechanical properties. Existing investigations $[4,37,14]$ consisted in applying a tensile loading on flat samples of arterial material, revealing in partic1 ular the material's ability to undergo large strains and a characteristic stiffening 12 occurring above a given tensile stress. In order to capture this complex mechani-

13 cal behavior and a potential anisotropy of the response, uniaxial tensile tests have 14 been performed independently in the axial and circumferential directions $[32,8]$, 15 and separately to the different layers of the composite structure of the arterial wall $[22,46]$. In parallel to this macroscopic characterization of the mechanical 17 properties, the microstructure of the vascular wall has been extensively analyzed by different microscopy techniques. In particular scanning electron microscopy allowed a morphological analysis of the arterial microstructure at the micron scale

$20 \quad[20,55,12,35]$. As for dynamic 2 nd harmonic microscopy, it enabled simultaneous 21 image acquisition and optical analysis of collagen fiber orientation, by means of 22 the collagen's optical property of second harmonic generation in the presence of 

46 particular bundle waviness and orientation $[6,40,28,54]$.

an intense laser beam coupled to a polarizer $[43,48,26]$. Confocal microscopy and multiphoton microscopy enabled live imaging with independent emissions signals corresponding to elastin and collagen [50] providing a 3D point of view on morphological characteristics of the vascular wall, and allowing the evaluation of volume fractions of the different components of the microstructure [31]. These techniques revealed the morphology of each concentric layer (intima, media, adventitia). Concerning the intima, it is composed of endothelial cells, oriented longitudinally [37]. As for the media, it is composed principally of circumferentially oriented smooth muscle cells and collagen fibers embedded in an elastin network $[56,10,53,13,16]$. Finally, the adventitia is composed of thick collagen bundles and of a net of helically oriented elastic fibers [6]. Recently, a special attention has been dedicated to further characterize collagen and elastin fibers, for instance by measuring the waviness of adventitial collagen [36], fiber segment length and the radially-connecting fiber density in the media $[49,29]$. Also, several studies started investigating the link between the arterial tissue's macroscopic mechanical response and the associated rearrangements of its microstructure, by coupling mechanical testing with live microscopy. The latter studies confirmed the load-bearing properties of collagen fibers and revealed a progressive morphological rearrangement under load, namely decrimping and reorientation of the collagen fibers in the direction of the load, as well as the subsequent stiffening of the material's response $[48,45,18,54]$. Those tests consisted in the application of uniaxial tension on flat samples [52,21,38], biaxial tension on flat samples $[39,23,47,27,28,7]$, or tension-inflation on cylindrical samples $[19,58,17]$. The observed morphological rearrangements concerned in

As a conclusion, extensive characterizations of both the mechanical layerspecific anisotropic behavior of the arterial wall, and the load-free microstructure morphology exist. Moreover, studies aiming at the characterization of load-induced microstructure rearrangements also exist and come up with advanced insights into the coupling between macro-mechanical response and tissue microstructure rear- 
rangements. However neither the underlying microscopic mechanisms governing the load-induced microstructure rearrangements nor the inter-layer differences in the rearrangements of the collagen and elastin networks have been characterized.

This paper is devoted to bridge these gaps in knowledge, by answering the following questions: are the well-known morphological changes of the vascular tissue under load (uncrimping, realignment) dependent on the direction of the load, on the vascular layer, and on the constituent under consideration? Are these morphological changes governed exclusively by the rule of affine transformations [5, $25] ?$

\section{Materials and methods}

2.1 Sample preparation

Seven carotid arteries (Fig. 1(a)) were harvested from healthy male New Zealand White rabbits, weighing $3 \mathrm{~kg}$ approximately. Excisions were realized at the Veterinary Campus of the Université de Lyon (VetAgro Sup, Marcy l'Étoile, FR). Rabbit cadavers, previously sacrificed under compliance with the NIH Guide for Care and Use of Laboratory Animals, were kindly provided by Centre Lago (Vonnas, FR). The length of each carotid was measured in vivo and ex vivo, i.e. immediately after harvesting (Table 1 - columns 2 and 3), in order to evaluate the in vivo pre-stretch condition (computed as the ratio of the ex vivo length to the in vivo length, Table 1 - column 4). The arteries were immediately frozen at $-20{ }^{\circ} \mathrm{C}$ until the day of the experimental tests and unfrozen in a bath of phosphate-buffered saline (10x PBS, pH 7.1) at ambient temperature $\left(24^{\circ} \mathrm{C}\right) .10 \mathrm{~mm}$ long cylindrical portions were excised from the arteries and longitudinally cut open, with a resulting width of approximately $5 \mathrm{~mm}$. For each artery, a $0.5 \mathrm{~mm}$ long ring (Fig. 1(b)) was also extracted for optical measurement of the arterial thickness (Table 1 - last column). This resulted in a cross-sectional area of $0.5 \pm 0.1 \mathrm{~mm}^{2}$. The rectangular strips were cut into dogbone shapes [21] aligned along the three following in-plane 
directions: circumferential, longitudinal, and an intermediate direction making a

$45^{\circ}$ angle with respect to the longitudinal direction (see Fig. 2(a) for a sketch of the sample preparation). In the following, this intermediate direction will be referred to as the diagonal direction. Twelve samples (four in each orientation group) were dedicated to mechanical testing coupled to multiphoton microscopy, while 6 additional samples (two in each orientation group) were dedicated to mechanical testing alone.

\subsection{Tensile test}

A screw-driven high precision tensile machine (Deben ${ }^{\circledR}$ Microtest tensile/compression stage) was used for uniaxial tensile tests, with a $150 \mathrm{~N}$ capacity load cell (Fig. 1(c)). The load cell signal provided a $0.01 \mathrm{~N}$ precision with satisfactory stability. The two heads of the tensile device moved in opposite directions, while the force and the displacement were recorded. Particular care was taken to ensure that the protocol for sample fixation limited the risk of inducing any pre-strain or pre-stress in the sample. Each sample underwent quasi static triangular preconditionning. According to the choice made by Hill et al. [21], a displacement-speed control was applied a rate of $0.5 \mathrm{~mm} \cdot \mathrm{min}^{-1}$, corresponding to a relative elongation speed of $0.2 \mathrm{~min}^{-1}$, with fixed target tensile force equal to $1 \mathrm{~N}$. During the mechanical test, the sample was continuously immerged in PBS at a constant ambient temperature of $24{ }^{\circ} \mathrm{C}$. After preconditioning, four measures of the sample width in the unloaded configuration were recorded using a caliper and averaged [21]. An image of the harvested ring of the artery was taken using a macro optic objective (Nikon D7200 ${ }^{\circledR}$ optical camera equipped with Nikon AF-S VR Micro-Nikkor ${ }^{\circledR}$ optical $105 \mathrm{~mm} \mathrm{f} / 2.8 \mathrm{G}$ IF-ED lens). The caliper in $0.5 \mathrm{~mm}$ opening position was placed next to the sample ring in order to caliber the pixel size and subsequently measure the thickness of the arterial wall. The measures of the sample width and thickness allowed the computation of the reference cross-sectional area $A_{0}$ (under zero load, after preconditioning) and of the first Piola-Kirchhoff (engineering) stress 
[21] $\sigma_{P K}=F / A_{0}$, where $F$ is the displacement-dependent measure of the tensile

force. The stretch was computed from the actual and reference inter-clamp length of the strip (respectively $l$ and $l_{0}$ ) as: $\lambda=l / l_{0}$. The reference length of the strip was defined as the interclamp length of the strip after preconditioning.

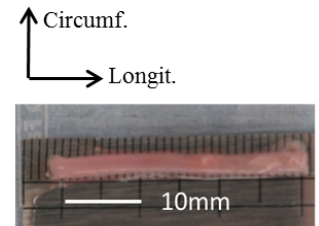

(a)

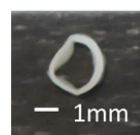

(b)

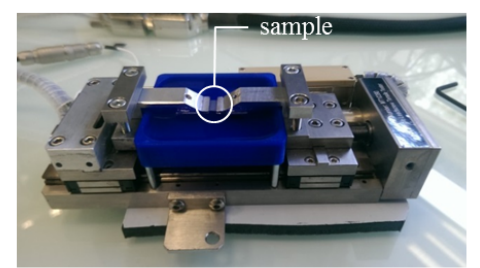

(c)

Fig. 1 (a) Excised carotid artery from a New Zealand White rabbit; (b) Cross-sectional ring of the artery for optical thickness measurement; (c) Tensile machine.

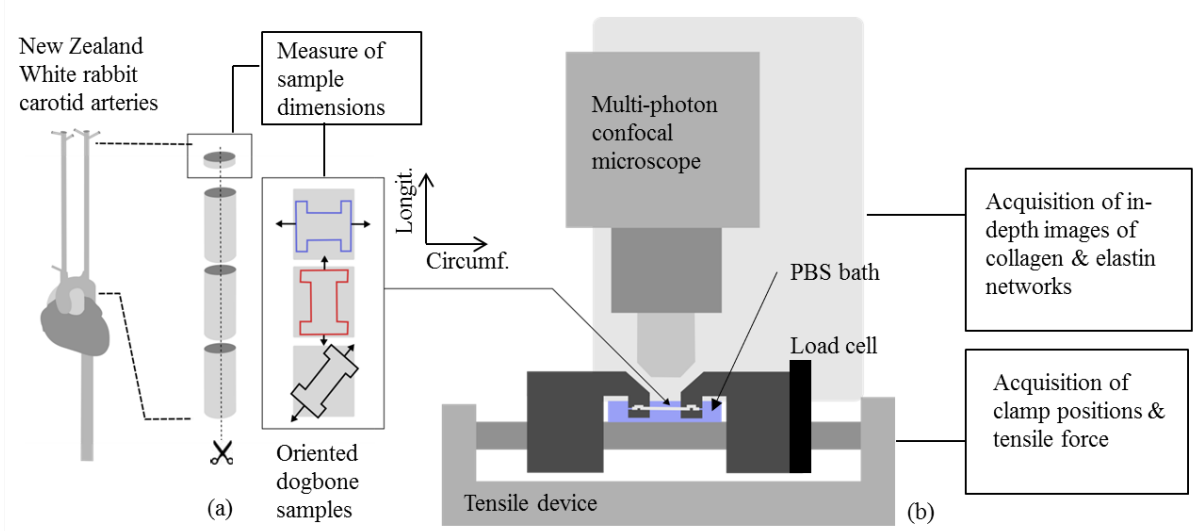

Fig. 2 (a) Schematic representation of the vascular tissue samples, prepared from cut-open cylindrical portions of New Zealand White rabbit carotid arteries; (b) Schematic representation of the experimental setup composed of a tensile machine coupled to a multiphoton microscope. 


\subsection{Image analysis}

The image stacks representing collagen and elastin were analyzed in terms of spatial orientation of the fibrous networks. Previous studies have investigated the transmural angle (radial direction) of the fibers and showed that it is negligible in comparison to the in-plane angle $[38,36,40]$. They have also showed that the most relevant morphological changes in the microstructure occur in the circumferential and longitudinal directions. Subsequently, we focused in the following on the anal- 
ysis of the in-plane angles of the fibrous networks. Prior to the analysis of relative angle densities, the image stacks were pre-processed so that they could represent the true tissue morphology on two-dimensional images while enabling liable angle density extraction. To this aim, the stack portion representing the adventitial layer of the vascular wall was subdivided into three equally thick sublayers (20-30 $\mu \mathrm{m})$ and each sublayer was projected orthogonally onto a single image using a maximum intensity projection algorithm. The choice of three sublayers allowed to achieve an optimum between image pixel density and fiber trans-mural continuity (due to non-zero transmural angles which could cause bias in the angle density analysis). Pre-processing of the media stack consisted in a projection in the thickness of one representative lamella $(5-10 \mu \mathrm{m})$, after verification of the equivalent morphologies of the different lamellae. The resulting set of $2 \mathrm{D}$ images used for the analysis of the fiber angle density consisted in three images of the adventitial collagen, three images of the adventitial elastin, one image of the medial collagen and one image of the medial elastin (Fig. 3(a)). The relative angle densities of the fibrous networks seen on each of these images were analyzed by combining wedge-shape integration of the Fourier power spectrum [2,42,41] (Fig. 3(b)) and a custom method for the extraction of local density maxima with their associated dispersions (Fig. 3(c)). Local density maxima were determined by a standard peak detection algorithm. The associated dispersions were evaluated by first determining an arbitrary density threshold that represented a cumulative percentile of total fiber angles extracted from the image, and secondly by reading out angle values corresponding to this threshold. Explicitly, let $\theta$ represent a local orientation angle, and $d(\theta)$ the angle density of the analyzed image; the angle density threshold $p$ is the angle density below which the fraction $\alpha_{p}=0.8$ of the total fibers is counted, i.e. the solution of the following equation:

$$
\alpha_{p}=\frac{\int_{0}^{\pi} \min (d(\theta), p) d \theta}{\int_{0}^{\pi} d(\theta) d \theta}=0.8
$$


We evaluated corresponding angles about each detected local maximum in order to extract related relative dispersions (Fig. 3(c)).

2.5 Predicting the amplitude of fiber rotation by affine reorientation

The assumption of affine reorientation considers that the fibers are continuously embedded in their surrounding matrix and are therefore constrained to the same deformation gradient as the matrix $[3,5,25]$. For a uniaxial tensile loading, the fiber orientations $\theta$ can be computed as a function of the initial angle $\theta_{0}$ and deformation stretch $\lambda$ (Fig. 3(d)) by:

$$
\theta=\arctan \left(\lambda \tan \theta_{0}\right)
$$

The angle values predicted by Equation (2) are compared to the experimentally measured angles in order to analyze to which extent this affine reorientation can predict true fiber network reorientations. In particular, the adventitial collagen bundles showed, in the load-free state, a dense crimped configuration for which it was difficult to extract global orientations of the bundles using traditional image processing techniques (Fourier analysis, local gradients, ...). In order to avoid a subsequent imprecision of the analysis, a specific method was designed to isolate the global fiber rotation mechanism from the uncrimping mechanism, and to test the hypothesis of affine kinematics only on the global reorientation of the fibers. To this aim, we considered a deformation scenario which starts in the diagonally deformed state and reaches the circumferentially and longitudinally deformed states in two sequenced steps: diagonal unloading followed by circumferential or longitudinal loading. Accordingly, only deformed configurations with straight collagen bundles needed to be processed for fiber angle analysis. Prediction errors were computed by dividing the difference between experimental and predicted fiber angles by the experimental fiber angle. 


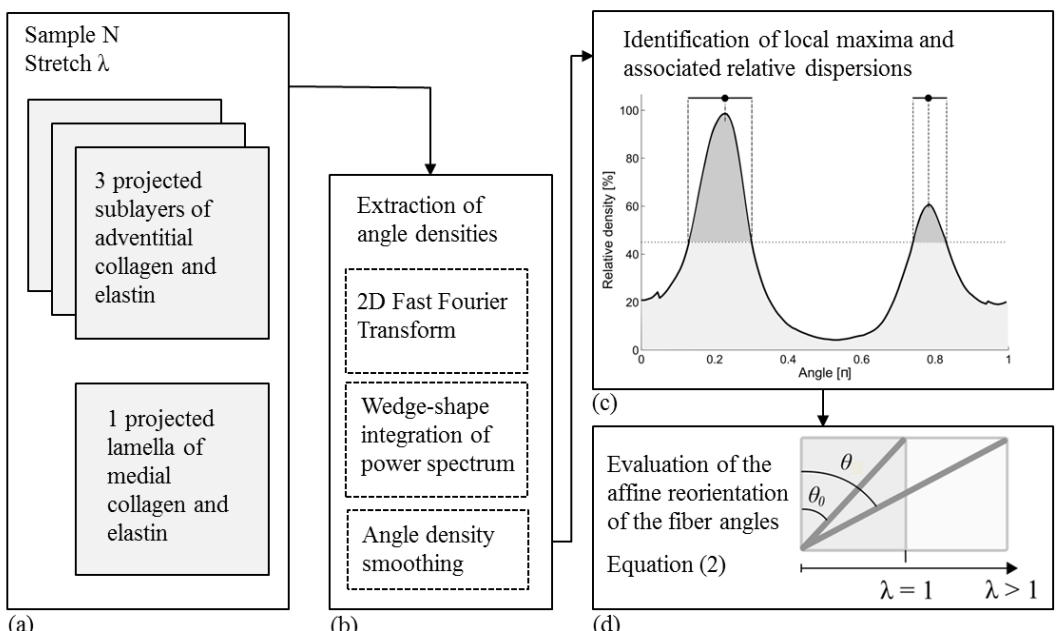

Fig. 3 Summary of the image analysis method. (a) Partial z-projections of adventitial and medial microstructure image stacks; (b) Fast Fourier 2D analysis of microstructure images; (c) Local maxima dectection and associated relative dispersions evaluation on smoothed fiber angles density function; (d) Analytical computation of affine fiber reorientation.

\section{Results}

\begin{tabular}{lllll}
\hline Specimen & $\begin{array}{l}\text { Ex vivo } \\
\text { length } \\
{[\mathrm{mm}]}\end{array}$ & $\begin{array}{l}\text { In vivo } \\
\text { length } \\
{[\mathrm{mm}]}\end{array}$ & $\begin{array}{l}\text { In vivo } \\
\text { stretch } \\
{[-]}\end{array}$ & $\begin{array}{l}\text { Thickness } \\
{[\mathrm{mm}]}\end{array}$ \\
\hline 1 & 4.3 & 7.1 & 1.6 & 0.18 \\
2 & 4.1 & 6.9 & 1.6 & 0.17 \\
3 & 4.3 & 7.0 & 1.6 & 0.19 \\
4 & 4.2 & 6.9 & 1.6 & 0.16 \\
5 & 4.3 & 7.5 & 1.7 & 0.18 \\
6 & 4.0 & 6.5 & 1.6 & 0.18 \\
7 & 3.9 & 6.6 & 1.7 & 0.19 \\
\hline
\end{tabular}

Table 1 Geometrical measurements of the harvested carotid arteries.

3.1 Macromechanical response of the arterial tissue

Engineering stress evaluated against stretch revealed a macroscopic anisotropy of of the samples starting at different stretch values depending on the direction of the load (Fig. 4(a)); (ii) the estimated elastic moduli in the final loading regime 
(Figure 4(b)-middle), and (iii) the stretch values when reaching $0.5 \mathrm{MPa}$ (Figure 4(b)-right). In particular, the mean estimated elastic moduli in the final loading regime was $50 \%$ higher in the circumferential direction than in the longitudinal direction; and $20 \%$ higher in the circumferential direction than in the diagonal direction. Accordingly, the mean stretch when reaching 0.5 MPa was $20 \%$ lower in the circumferential direction than in the longitudinal direction; and $15 \%$ lower than in the diagonal direction. Concerning the estimated elastic moduli in the initial loading regime (Figure 4(b)-left), the comparison of mean values showed lower differences between the sample families as their standard deviation amounts to $8 \%$. Comparing the measured in vivo stretches to the longitudinal mechanical response (Table 1), the carotid were subjected in vivo to a longitudinal stress of about 0.1 MPa, in the soft region of the curve (Fig. 4 - red curves).

3.2 Microstructure morphological rearrangements under uniaxial load

Fibrous networks of the arterial microstructure revealed varying morphologies depending on the biological constituent (elastin, collagen), on the considered arterial layer (adventitia, media), and on the direction of the applied uniaxial load (Fig. 5). Fig. 5(a) shows the microstructure of the unloaded arterial tissue, and Fig. $5(\mathrm{~b})(\mathrm{c})(\mathrm{d})$ show the microstructure under the three considered loading conditions, which corresponded to the same uniaxial force of 0.8 N. For each constituent of the imaged microstructure, we provide on Fig. 6 a polar representation of fiber orientations (local maxima of angle density with associated dispersions, see section 2.4 for more details) with the relative elongation $\lambda$ and the fiber angle $\theta$ as polar coordinates. The angles $\pi / 2,3 \pi / 4$, and $\pi$ represent the circumferential, diagonal, and longitudinal directions respectively.

Adventitial collagen was organized in thick bundles showing a crimped morphology in the load-free state (Fig. 5(a) - upper left). The analysis of in-plane fiber angles (Fig. 6(a)) suggested a preferred direction close to the longitudinal direction, with an important dispersion $( \pm \pi / 6)$ due to bundle direction variabil- 


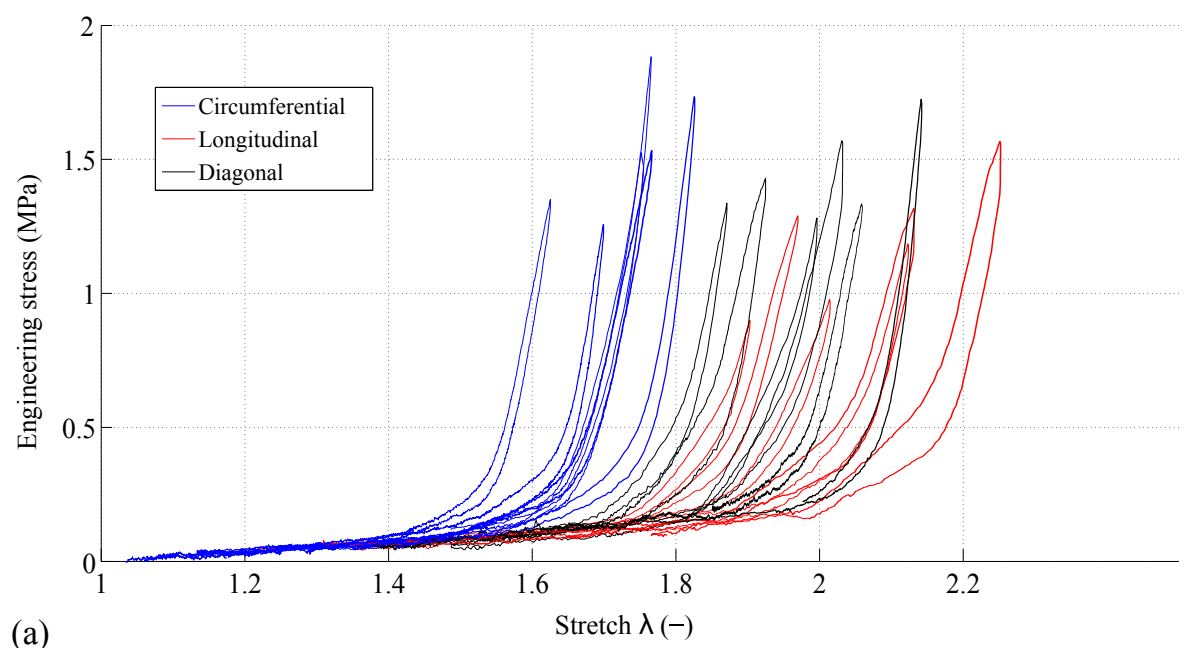

(a)

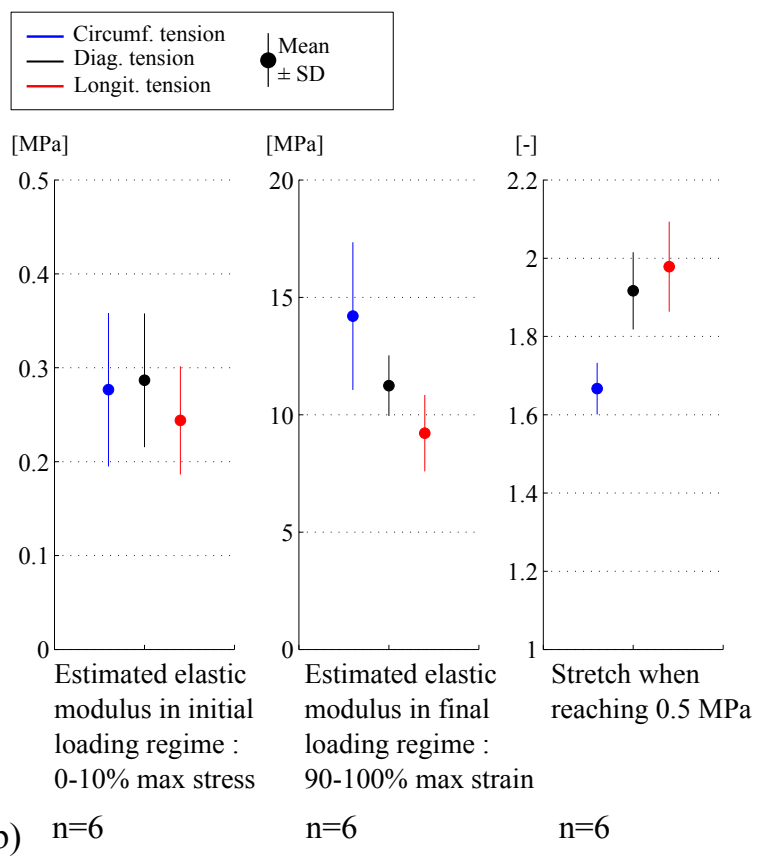

Fig. 4 (a) Macroscopic mechanical response: engineering stress PK v.s. stretch plotted for the three loading directions (blue: circumferential, black: diagonal, red: longitudinal); (b) Statistical representations of the stiffness before (left) and after (middle) collagen engagement, and stretch when reaching $0.5 \mathrm{MPa}$ (right); $\mathrm{n}$ stands for the number of investigated samples 

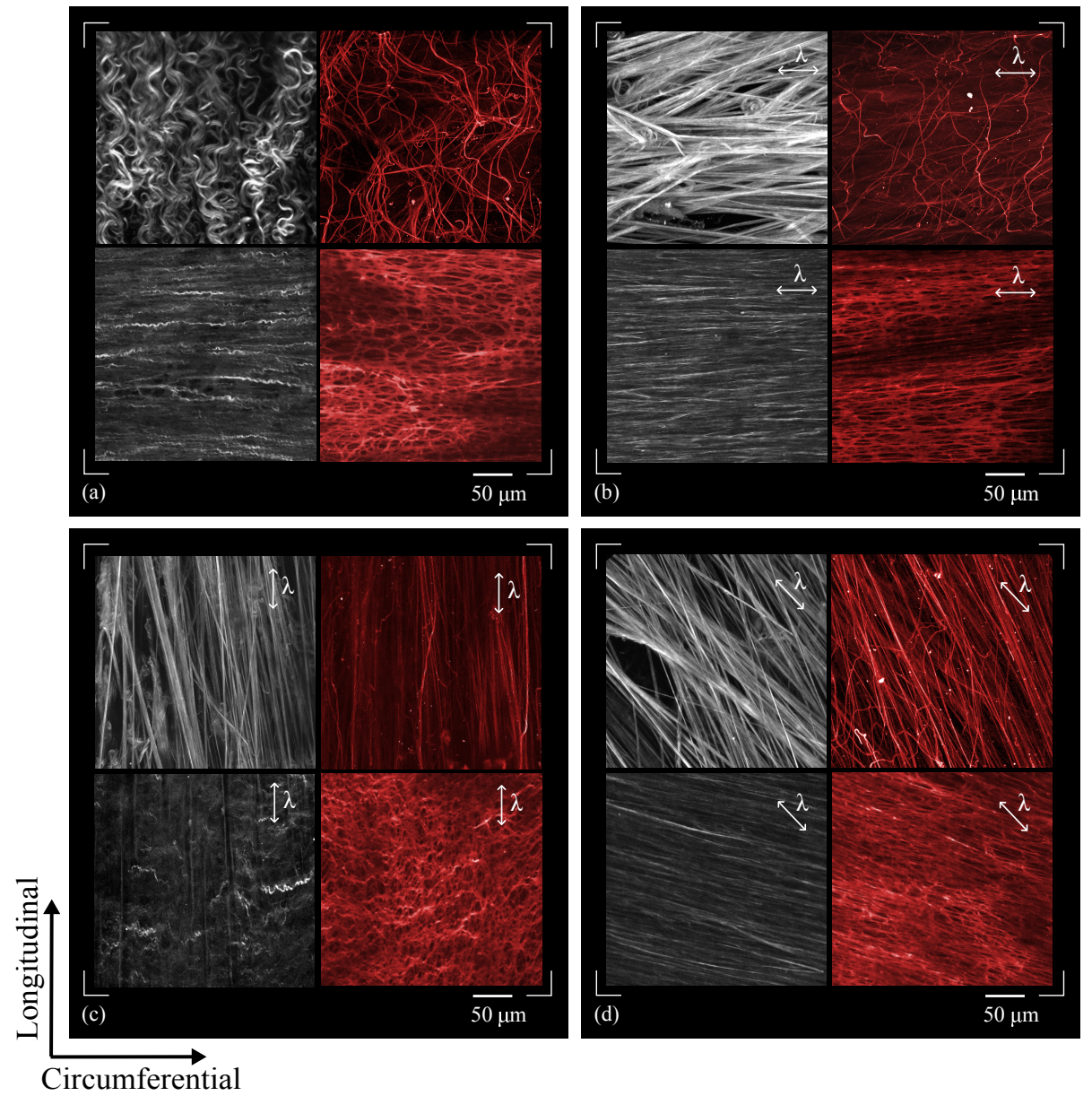

Fig. 5 Arterial microstructure imaged under multiphoton microscope. (a) Load-free state, (b) circumferential load ( $\mathrm{F}=0.8 \mathrm{~N}, \lambda=1.7)$, (c) longitudinal load $(\mathrm{F}=0.8 \mathrm{~N}, \lambda=2.1)$, and (d) diagonal load $(\mathrm{F}=0.8 \mathrm{~N}, \lambda=1.9)$. The upper row of each image represents the adventitia; the lower row represents the media. Collagen network is represented in grey (left), elastin network is represented in red (right).

ity and to the influence of crimping, which limited the liability of interpretation.

These collagen bundles underwent an uncrimping process and all appeared to align close to the direction of the load, whether its direction was circumferential (Fig. 5(b) - upper left), longitudinal (Fig. 5(c) - upper left) or diagonal (Fig. 5(d) upper left). In the three deformed states, in-plane fiber orientations (Fig. 6(a)) showed identically limited dispersions $( \pm \pi / 12)$ around their respective local angle maximum. 


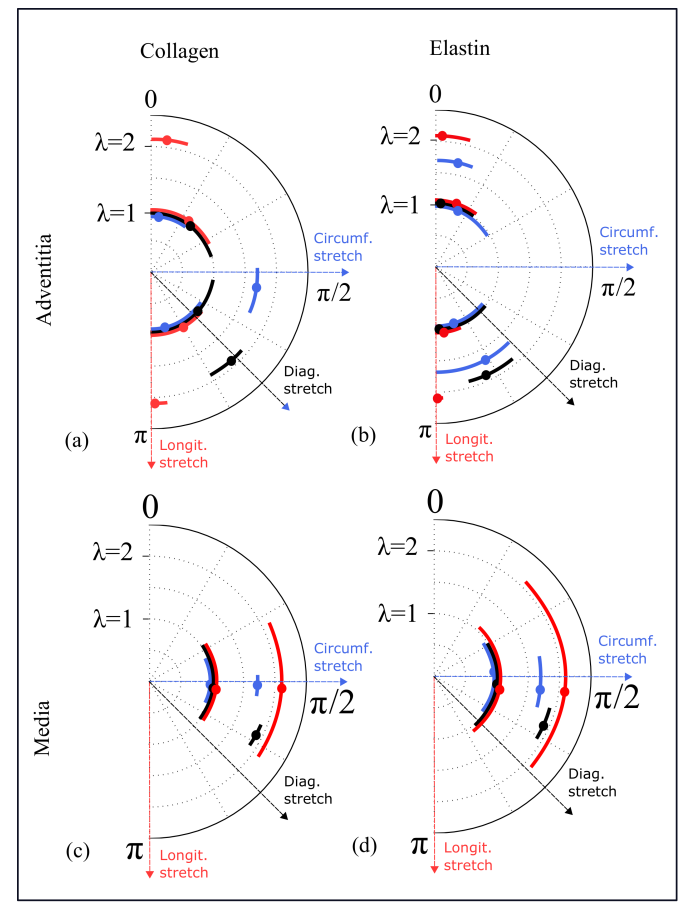

Fig. 6 Orientations of fiber networks represented with local angle density maxima and associated dispersions, for each constituent, layer, and load direction, under 0 and $0.8 \mathrm{~N}$ tensile load: (a) and (e) correspond to adventitial collagen, (b) and (f) to adventitial elastin, (c) and (g) to medial collagen, and (d) and (h) to medial elastin. The blue, black, and red colors correspond to the circumferential $(\lambda=1.7)$, diagonal $(\lambda=1.9)$, and longitudinal $(\lambda=2.1)$ directions respectively. Represented network orientations correspond to the mechanical deformations of samples 1-3, tested respectively in the circumferential, diagonal and longitudinal directions.

Adventitial elastin took the form of a low-density meshwork, composed of thin fiber segments showing a preferred longitudinal direction with variously oriented transverse segments (Fig. 5(a) - upper right). The analysis of in-plane fiber angles at rest (Fig. 6(b)) confirmed the longitudinal orientation, with an important dispersion $( \pm \pi / 6)$ due to the variously oriented transverse fiber segments. Under load, the longitudinally oriented fiber segments underwent limited morphological changes: when the loading direction was circumferential (Fig. 5(b) - upper right), the global orientation of the meshwork did not change significantly (a maximum of $\pi / 6$ rotation - Fig. $6(\mathrm{~b}))$, although a spreading of the meshwork was noticeable (compare Fig. 5(a) - upper right and 5(b) - upper right). Almost unchanged dispersions around local density maxima (Fig. 6(b)) confirmed this limited morphological 
change. When the load was applied longitudinally (Fig. 5(c) - upper right), transverse segments aligned in the direction of the load, while the fibrous meshwork remained oriented in the longitudinal direction, which led to an unchanged angle density (Fig. 6(b)). When the load was applied in the diagonal direction (Fig. 5(d) - upper right), the meshwork underwent a limited reorientation towards the loading direction (a maximum rotation of $\pi / 6$ and angle dispersions reduced by a factor 2 - Fig. 6(b)), with transverse segments aligning with the principal segments orientations.

Medial collagen did not agglomerate into variously oriented bundles as in the adventitia, but took the form of thin parallel fibers distinctively oriented in the circumferential direction (Fig. 5(a) - lower left) and showed a higher crimping frequency. Its morphology underwent limited changes under uniaxial load: when the loading direction was circumferential (Fig. 5(b) - lower left), the fibers uncrimped and remained aligned in the circumferential direction (Fig. 6(c)). When the loading direction was diagonal (Fig. 5(d) - lower left), the fibers uncrimped - with a decrease of the dispersion of local angles by a factor 2 (Fig. 6(c)) - and underwent a limited realignment in the loading direction - below $\pi / 6$ rotation for the density maximum of local angles (Fig. 6(c)). When the load was longitudinal (Fig. 5(c) - lower left), i.e. perpendicular to the network direction, the fibers did not align in the loading direction and their crimping increased, as shown by the angular dispersion increased by a factor 2 (Fig. 6(c)).

The medial elastin morphology was different from the one of adventitial elastin: at rest (Fig. 5(a) - lower right), it took the form of a dense, structured meshwork preferentially oriented in the circumferential direction. Under load, it underwent limited morphology changes, namely partial alignment of transverse fiber segments in the loading direction, when the loading direction was circumferential (Fig. 5(b) - lower right) or longitudinal (Fig. 5(c) - lower right); the maxima of angle density remained unchanged, while the angular density dispersion decreased by a factor 
2 (Fig. 6(d)); and a limited global reorientation when the loading direction was diagonal, with rotation of the angle density maximum below $\pi / 6$.

\subsection{Prediction of reorientations using an affine model}

Table 2 presents the experimental values of local angle density maxima with the upper and lower angle bounds corresponding to the evaluated dispersions, together with the theoretical angles computed by applying the affine reorientation (Equation (2)) to the local angle maxima. The represented results in Table 2 refer to the testing of samples 1 to 3 , respectively in the circumferential, diagonal and longitudinal directions. Fig. 7 presents a statistical synthesis of fiber angles under uniaxial load, together with the theoretical angles computed by applying the affine reorientation (Equation (2)) to the local angle maxima. The represented results refer to the four sample groups tested in the three aforementioned directions (samples 1 to 12 ). Results show that the affine model predicted well the reorientation of the medial microstructure (collagen and elastin) as well as of the adventitial elastin, with prediction errors ranging from 1 to $7 \%$ (see Table 2 and compare black and white symbols in Fig. 7) and a limited variability of fiber orientation between the analogous samples (Fig. 7). Concerning adventitial collagen, for which we illustrated the ability to reorient along the different tested loading directions (Fig. 5, Fig. 6), the affine model largely under-predicted the fiber reorientation, as proven by the high prediction error (12 to $25 \%$, see Table 2 ), observed independently on the analogous samples (Fig. 7, upper left).

\section{Discussion}

Our results represent an original insight into the morphological changes that occur in the arterial tissue microstructure under variously oriented uniaxial loads. The experiments were performed on rabbit carotid arteries and focused on collagen and elastin networks of the media and of the adventitia. Our results suggest that 


\begin{tabular}{lllll} 
& & (a) Initial & (b) Final 1 & (c) Final 2 \\
Group 1 : samples 1-3 & & $\lambda_{\text {diag }}=1.9 \pm 0.1$ & $\lambda_{\text {circ }}=1.7 \pm 0.05$ & $\lambda_{\text {long }}=2.1 \pm 0.2$ \\
\hline Collagen - adv. & Experimental $[\pi]$ & $0.76_{-0.02}^{+0.07}$ & $0.55_{-0.06}^{+0.07}$ & $0.97_{-0.05}^{+0.03}$ \\
& Affine prediction $[\pi]$ & - & 0.69 & 0.85 \\
& Prediction error $[\pi]$ & - & $\mathbf{2 5 \%}$ & $\mathbf{1 2 \%}$ \\
\hline Elastin - adv. & Experimental $[\pi]$ & $0.86_{-0.09}^{+0.04}$ & $0.83_{-0.13}^{+0.12}$ & $0.98_{-0.01}^{+0.02}$ \\
& Affine prediction $[\pi]$ & - & 0.89 & 0.97 \\
& Prediction error & - & $\mathbf{7 \%}$ & $\mathbf{1 \%}$ \\
\hline Collagen - med. & Experimental $[\pi]$ & $0.61_{-0.03}^{+0.04}$ & $0.51_{-0.02}^{+0.02}$ & $0.52_{-0.17}^{+0.16}$ \\
& Affine prediction $[\pi]$ & - & 0.52 & 0.54 \\
& Prediction error & - & $\mathbf{2 \%}$ & $\mathbf{4 \%}$ \\
\hline Elastin - med. & Experimental $[\pi]$ & $0.60_{-0.06}^{+0.07}$ & $0.53_{-0.08}^{+0.03}$ & $0.53_{-0.21}^{+0.22}$ \\
& Affine prediction $[\pi]$ & - & 0.50 & 0.51 \\
& Prediction error & - & $\mathbf{6 \%}$ & $\mathbf{4 \%}$ \\
\hline
\end{tabular}

Table 2 Evaluation of the predictive accuracy of affine reorientation. Fiber angles are provided for initial and final states of two deformation scenarii (Samples 1-3) : (a) to (b) and (a) to (c)

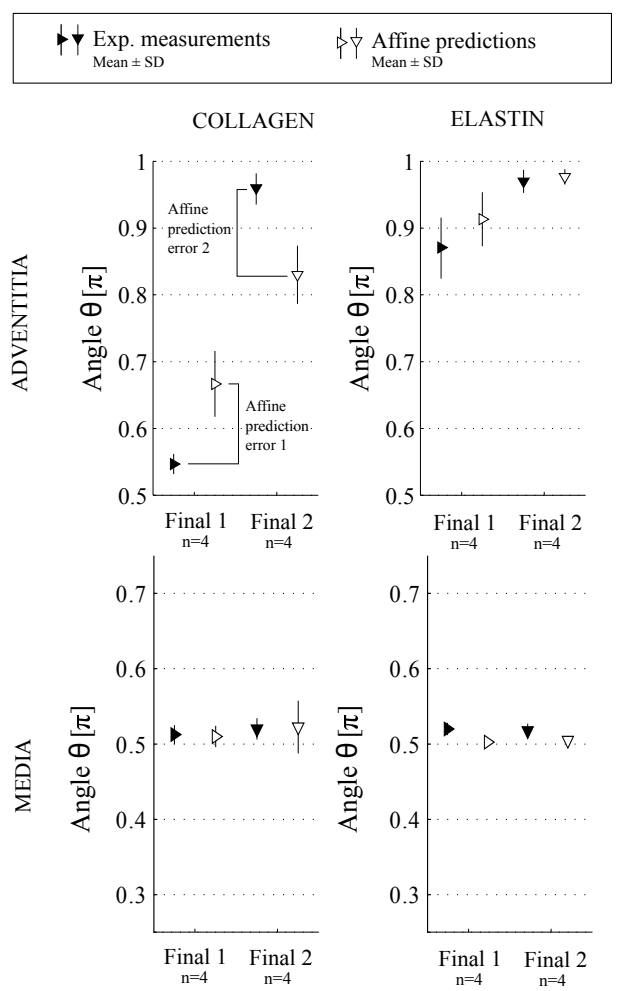

Fig. 7 Synthesis of the predictive accuracy analysis of affine reorientation. Final network orientations (Final state 1 and Final state 2) are averaged on $n=4$ samples under analogous deformation. Affine prediction errors in the case of adventitial collagen reorientation amount to $0.12 \pi$ and $0.13 \pi$ when reaching final states 1 and 2 respectively, corresponding to $22 \%$ and $14 \%$ error.

the arterial tissue is more compliant in the longitudinal direction, as seen by a delayed stiffening phase. This result is consistent with previous studies performed 
[11] and by applying uniaxial tension on flat samples of human ascending aortas

[8]. Conversely, this anisotropy has been shown contrary (stiffer tissue in the longitudinal direction) for human coronary arteries [33] and dog coronary arteries [34]. Indeed, no consensus has been gained up to now as regards the anisotropy of arteries, evidencing the complex interplay of load bearing contributions between the media and the adventitia. Recent results [22] show clearly that the media is stiffer in the circumferential direction, while the adventitia is stiffer in the longitudinal direction. These results are in good agreement with the orientation of the fiber networks in each layer, but do not permit to draw any conclusion on the overall anisotropy of the tissue. In order to further understand the origin of this complex anisotropy, the distribution of load bearing properties between the media and adventitia should be further characterized along with the study of microstructure rearrangements.

Concerning the arterial microstructure in the load-free state, our results illustrate the layer-specific morphologies of the collagen and elastin networks which are similar to previous investigations $[31,36]$. In the deformed configuration however, our results bring a novel comprehensive understanding of morphological changes occuring within the arterial microstructure. Under uniaxial tensile loadings, affine kinematics $[3,5]$ explain the reorientations of adventitial elastin, medial elastin, and medial collagen. This kinematically limited reorientation corresponds to a tight embedding of the fibers in their surrounding matrix, which could correspond to near-equivalent shear moduli of the fibers and of their surrounding matrix, or to the existence of molecular links which hinder further rotation of the inclusions within the matrix. Besides, these fibrous networks are unable to fully align in any of the presently applied uniaxial loading directions. Conversely, the adventitial collagen showed a remarkable potential to reorient in any of the applied loading directions (circumferential, longitudinal, diagonal). This reorientation is characterized by significant rotation amplitudes, that remain underestimated by affine reorientations. Although the protocols employed in the literature vary both 
in terms of characterization techniques (focusing on fiber decrimping [30] or on global fiber reorientation [24]) and in terms of applied mechanical loadings (uniaxial, equi-biaxial [30]), our result is consistent with previous studies investigating collagen fiber kinematics in other tissues like porcine aortic leaflets [3], bovine pericardium [3], tissue constructs [5] or human liver capsule [24] and suggests that other mechanisms are activated in the reorientation of adventitial collagen under load.

Several limitations stem from the differences existing between our testing protocol and the real in vivo conditions. First of all, we should comment on the fact that we have considered temperature conditions lower than in vivo conditions. Zemanek et al. [57] studied the influence of the testing temperature on the mechanical response of the arterial wall and showed that samples are stiffer at ambient temperature than at in vivo temperature. There is no consensus on the impact of sample freezing on the change in the mechanical response of the sample $[57,1,51,9]$. However in the present study, test temperature and preservation conditions were identical for all experiments, and allowed to perform an inter sample comparison of the stiffness change during loading or among diverse load directions. Also, to our best knowledge, there is no study on the influence of the temperature on the fiber rotation. Secondly, we have applied a quasi-static elongation speed, which cannot render the dynamic conditions of the pulsatile hemodynamic load seen in large vessels. However, given the small variations in the arterial pressure and the preferred helical orientation of the adventitial collagen bundles, the orientations of the latter are believed not to vary significantly during a cardiac cycle. The choice of a quasi-static loading was made so as to ensure that the mechanical response was independent of the strain rate, as well as to limit the relaxation effects that would impair repeatability of the results. We should also comment on the choice of directions and ranges of load that go beyond the physiological load directions and ranges. While tension-inflation or equi-biaxial protocols allow the simulation of near physiological loadings, the present protocol was applied to reach our objec- 
tive of comparing inter-layer and inter-constituent changes of the fiber networks within the microstructure subjected to diversified loading scenarii. Besides, the high stretches reached successively during preconditioning and imaging may have induced damage in the tissue, enhancing non-affine fiber rotation. However it is assumed that in this case, the increase of fiber rotation would have affected all imaged fibrous networks, not only adventitial collagen. Accordingly, we did not observe any sign of damage within the imaged tissue, on any fibrous network of the arterial wall.

Furthermore, it should be mentioned that what we call arterial microstructure in this work is incomplete from the biological point of view, as our multiphoton microscopy provided only images of elastin and collagen fibers, with a spatial resolution of the order of $1 \mu \mathrm{m}$. The analysis could be complemented by the characterization of the deformations and reorientations of non-cellular components under varying levels of smooth muscle tones, as the latter mechanism is known to play an active role in arterial adaptive plasticity during the in vivo pressure cycle. At a lower scale, our analysis could also be complemented by the description of collagen fibrils rearrangements within bundles during decrimping and during postdecrimping stretching. Moreover, the observed morphology changes during uniaxial tension in carotid samples should be compared to morphology changes occurring in identically deformed carotids from other species, with different smooth muscle, collagen and elastin content. To the authors' knowledge, identical deformation protocol has not been yet applied to samples of other arteries or other species carotids. However, and to engage the discussion, the recent studies of Keyes et al. [27] on mouse aortas, showed that adventitial collagen could undergo high amplitude rotations under pressurization (up to $\pi / 3$ ), while medial networks kept their orientation unchanged. On the contrary, the results of Chen et al. [7], performed on porcine coronary arteries, illustrated under flat biaxial tension the adventitial collagen's decrimping mechanism but a limited rotation potential. Nevertheless, our analysis was intended as a first step, focusing on the major load-bearing com- 
ponents of the arterial wall. Furthermore, a nano-scale imaging resolution could investigate the influence of nanoscale aldehyde cross-links on the observed morphology rearrangements. Such an analysis would give biological foundations to the observed reorientations, explaining why several fibers can encounter large rotations whilst others are restricted to small rotations.

Finally, the level of representativeness of the microscopy images with associated angle distributions of collagenous and elastic networks should be commented. In particular concerning automated angles extraction, the Fast Fourier Transform algorithm applied on adventitial collagen at rest (high density crimped bundles) provided information about local angles but did not allow the extraction of global collagen bundle orientation. In order to capture full morphological rearrangements of adventitial collagen under load, a specific method should be developed so as to analyze collagen orientation at different scales: crimping scale (10 $\mu \mathrm{m})$ and global bundle scale $(100 \mu \mathrm{m})$. With the objective of comparing true reorientations with affine predictions, we here overcame this image processing issue by comparing only deformed configurations, for which the orientations at crimping scale and at global bundle scale are identical.

\section{Conclusion and outlooks}

We have shown that arterial microstructure presents inter-layer differences between collagen network and elastin network morphologies. These inter-layer differences concern in particular the ability to rearrange under variously oriented uniaxial loads. We have shown that even though the fibers of the vascular microstructure undergo the same macroscopic deformation, they rearrange in their own specific way, and differently from one vascular layer to the other. This finding brings additional insight for current multi-scale model formulations which take into account specific kinematics of arterial collagen and its relation to the surrounding matrix $[44,15]$. It also confirms the need to consider the arterial wall as a composite structure composed of different mechanically relevant layers with different mechanical 
properties [22]. Our results also suggest the need to further investigate the underlying mechanisms that govern adventitial collagen rearrangement, in particular uncrimping and post-uncrimping fiber rotation. Given that the affine reorientation underestimates real fiber rotation of adventitial collagen, it appears interesting to propose a micromechanical origin to collagen fiber rotation. In particular, we hypothesize that the macroscopic strain concentrated in a highly heterogeneous microstructure induces a heterogeneous field of displacement gradients, itself generating microscopic spin that can be important if the shear moduli of the fibers and of the matrix are significantly different. These spins may induce material rotation within the structure which superimposes with affine reorientation. Future multi-scale models could test this hypothesis by accounting for the existence of local spin vectors, promoting fiber rotations.

Acknowledgements This work was supported by the ARC 2 "Bien-être et vieillissement" research program of the Auvergne-Rhône-Alpes region (FR), with financial contributions from ERC consolidator grant "Biolochanics" and CNRS UMR 5513 research group. The authors thank Prof. Eric Viguier (VetAgro Sup, Université de Lyon, FR), Dr. Caroline Boulocher (VetAgro Sup, Université de Lyon, FR) and Mr. Fabrice Desplanches (Centre Lago, Vonnas, FR) for their help in provision and excision of arterial specimen. Authors also thank the IVTV (ANR-10-EQPX-06-01) team and especially Ing. Ophélie Pollet for her support during the imaging process, as well as Dr. Damien Constant (Ecole Centrale Lyon, FR) and Mr. Lionel Charles (Ecole Centrale Lyon, FR) for their technical contribution in the preparation of the mechanical setup.

\section{References}

1. Adham, M., Gournier, J.P., Favre, J.P., De La Roche, E., Ducerf, C., Baulieux, J., Barral, X., Pouyet, M.: Mechanical characteristics of fresh and frozen human descending thoracic aorta. Journal of Surgical Research 64(1), 32-34 (1996)

2. Ayres, C., Jha, B.S., Meredith, H., Bowman, J.R., Bowlin, G.L., Henderson, S.C., Simpson, D.G.: Measuring fiber alignment in electrospun scaffolds: a user's guide to the 2D Fast Fourier Transform approach. Journal of Biomaterials Science, Polymer Edition 19(5), 603-621 (2008) 
3. Billiar, K., Sacks, M.: A method to quantify the fiber kinematics of planar tissues under biaxial stretch. Journal of Biomechanics 30(7), 753-756 (1997)

4. Burton, A.C.: Relation of structure to function of the tissues of the wall of blood vessels. Physiological Reviews 34(4), 619-642 (1954)

5. Chandran, P.L., Barocas, V.H.: Affine versus non-affine fibril kinematics in collagen networks: theoretical studies of network behavior. Journal of Biomechanical Engineering 128(2), 259-70 (2006)

6. Chen, H., Liu, Y., Slipchenko, M.N., Zhao, X., Cheng, J.X., Kassab, G.S.: The layered structure of coronary adventitia under mechanical load. Biophysical Journal 101(11), 2555-2562 (2011)

7. Chen, H., Slipchenko, M.N., Liu, Y., Zhao, X., Cheng, J.X., Lanir, Y., Kassab, G.S.: Biaxial deformation of collagen and elastin fibers in coronary adventitia. Journal of Applied Physiology 115(11), 1683-1693 (2013)

8. Choudhury, N., Bouchot, O., Rouleau, L., Tremblay, D., Cartier, R., Butany, J., Mongrain, R., Leask, R.L.: Local mechanical and structural properties of healthy and diseased human ascending aorta tissue. Cardiovascular Pathology 18(2), 83-91 (2009)

9. Chow, M.J., Zhang, Y.: Changes in the mechanical and biochemical properties of aortic tissue due to cold storage. Journal of Surgical Research 171(2), 434-442 (2011)

10. Clark, J.M., Glagov, S.: Transmural organization of the arterial media. The lamellar unit revisited. Arteriosclerosis, Thrombosis, and Vascular Biology 5(1), 19-34 (1985)

11. Cox, R.: Anisotropic properties of the canine carotid artery in vitro. Journal of biomechanics 8(5), 293-300 (1975)

12. Denk, W., Horstmann, H.: Serial block-face scanning electron microscopy to reconstruct three-dimensional tissue nanostructure. PLoS Biology 2(11) (2004)

13. Dingemans, K.P., Teeling, P., Lagendijk, J.H., Becker, A.E.: Extracellular matrix of the human aortic media: an ultrastructural histochemical and immunohistochemical study of the adult aortic media. The Anatomical Record 258(1), 1-14 (2000)

14. Duprey, A., Khanafer, K., Schlicht, M., Avril, S., Williams, D., Berguer, R.: In vitro characterisation of physiological and maximum elastic modulus of ascending thoracic aortic aneurysms using uniaxial tensile testing. European Journal of Vascular and Endovascular Surgery 39(6), $700-707$ (2010)

15. Fan, R., Sacks, M.S.: Simulation of planar soft tissues using a structural constitutive model: finite element implementation and validation. Journal of biomechanics 47(9), 2043-2054 (2014)

16. Farand, P., Garon, A., Plante, G.E.: Structure of large arteries: Orientation of elastin in rabbit aortic internal elastic lamina and in the elastic lamellae of aortic media. Microvascular Research 73(2), 95-99 (2007) 
17. Ferruzzi, J., Vorp, D., Humphrey, J.D.: On constitutive descriptors of the biaxial mechanical behaviour of human abdominal aorta and aneurysms. Journal of the Royal Society, Interface 8(56), 435-450 (2011)

18. Genovese, K., Lee, Y.U., Lee, a.Y., Humphrey, J.D.: An improved panoramic digital image correlation method for vascular strain analysis and material characterization. Journal of the Mechanical Behavior of Biomedical Materials 27, 132-42 (2013)

19. Gleason, R.L., Gray, S.P., Wilson, E., Humphrey, J.D.: A multiaxial computer-controlled organ culture and biomechanical device for mouse carotid arteries. Journal of Biomechanical Engineering 126(6), 787-795 (2004)

20. Gross, J.: The structure of elastic tissue as studied with the electron microscope. The Journal of Experimental Medicine 89(6), 699-708 (1949)

21. Hill, M.R., Duan, X., Gibson, G.A., Watkins, S., Robertson, A.M.: A theoretical and nondestructive experimental approach for direct inclusion of measured collagen orientation and recruitment into mechanical models of the artery wall. Journal of Biomechanics 45(5), $762-771(2012)$

22. Holzapfel, G.A., Sommer, G., Gasser, C.T., Regitnig, P.: Determination of layer-specific mechanical properties of human coronary arteries with nonatherosclerotic intimal thickening and related constitutive modeling. American Journal of Physiology-Heart and Circulatory Physiology 289(5), H2048-H2058 (2005)

23. Humphrey, J.D., Wells, P.B., Baek, S., Hu, J.J., McLeroy, K., Yeh, A.T.: A theoreticallymotivated biaxial tissue culture system with intravital microscopy. Biomechanics and Modeling in Mechanobiology 7(4), 323-334 (2008)

24. Jayyosi, C.: Caractérisation mécanique et microstructurale du comportement à rupture de la capsule de glisson pour la prédiction du risque de lésions des tissus hépatiques humains. Ph.D. thesis, Université de Lyon 1 (2015)

25. Jayyosi, C., Coret, M., Bruyere-Garnier, K.: Characterizing liver capsule microstructure via in situ bulge test coupled with multiphoton imaging. Journal of the Mechanical Behavior of Biomedical Materials 54, 229-243 (2016)

26. Kabir, M.M., Inavalli, V.K., Lau, T.Y., Toussaint, K.C.: Application of quantitative second-harmonic generation microscopy to dynamic conditions. Biomedical optics express 4(11), 2546-2554 (2013)

27. Keyes, J.T., Borowicz, S.M., Rader, J.H., Utzinger, U., Azhar, M., Geest, J.P.V.: Design and demonstration of a microbiaxial optomechanical device for multiscale characterization of soft biological tissues with two-photon microscopy. Microscopy and Microanalysis $\mathbf{1 7}(02), 167-175(2011)$ 
28. Keyes, J.T., Lockwood, D.R., Utzinger, U., Montilla, L.G., Witte, R.S., Geest, J.P.V.: Comparisons of planar and tubular biaxial tensile testing protocols of the same porcine coronary arteries. Annals of Biomedical Engineering 41(7), 1579-1591 (2013)

29. Koch, R.G., Tsamis, A., D’Amore, A., Wagner, W.R., Watkins, S.C., Gleason, T.G., Vorp, D.A.: A custom image-based analysis tool for quantifying elastin and collagen microarchitecture in the wall of the human aorta from multi-photon microscopy. Journal of Biomechanics 47(5), 935-943 (2014)

30. Lee, C.H., Zhang, W., Liao, J., Carruthers, C.A., Sacks, J.I., Sacks, M.S.: On the presence of affine fibril and fiber kinematics in the mitral valve anterior leaflet. Biophysical journal 108(8), 2074-2087 (2015)

31. O’Connell, M.K., Murthy, S., Phan, S., Xu, C., Buchanan, J., Spilker, R., Dalman, R.L., Zarins, C.K., Denk, W., Taylor, C.A.: The three-dimensional micro- and nanostructure of the aortic medial lamellar unit measured using 3D confocal and electron microscopy imaging. Matrix Biology 27(3), 171-181 (2008)

32. Okamoto, R.J., Wagenseil, J.E., DeLong, W.R., Peterson, S.J., Kouchoukos, N.T., Sundt III, T.M.: Mechanical properties of dilated human ascending aorta. Annals of Biomedical Engineering 30(5), 624-635 (2002)

33. Papageorgiou, G., Jones, N.: Circumferential and longitudinal viscoelasticity of human iliac arterial segments in vitro. Journal of biomedical engineering 10(1), 82-90 (1988)

34. Patel, D.J., Janicki, J.S.: Static elastic properties of the left coronary circumflex artery and the common carotid artery in dogs. Circulation Research 27(2), 149-158 (1970)

35. Raspanti, M., Protasoni, M., Manelli, A., Guizzardi, S., Mantovani, V., Sala, A.: The extracellular matrix of the human aortic wall: ultrastructural observations by FEG-SEM and by tapping-mode AFM. Micron 37(1), 81-86 (2006)

36. Rezakhaniha, R., Agianniotis, A., Schrauwen, J.T.C., Griffa, A., Sage, D., Bouten, C.V.C., Van De Vosse, F.N., Unser, M., Stergiopulos, N.: Experimental investigation of collagen waviness and orientation in the arterial adventitia using confocal laser scanning microscopy. Biomechanics and Modeling in Mechanobiology 11(3-4), 461-473 (2012)

37. Richardson, P.D., Keeny, S.: Anisotropy of human coronary artery intima. In: Bioengineering Conference, 1989., Proceedings of the 1989 Fifteenth Annual Northeast, pp. 205-206. IEEE (1989)

38. Roy, S., Boss, C., Rezakhaniha, R., Stergiopulos, N.: Experimental characterization of the distribution of collagen fiber recruitment. Journal of Biorheology 24(2), 84-93 (2011)

39. Sacks, M.S.: Incorporation of experimentally-derived fiber orientation into a structural constitutive model for planar collagenous tissues. Journal of Biomechanical Engineering 125(2), 280-287 (2003) 
40. Schrauwen, J.T.C., Vilanova, A., Rezakhaniha, R., Stergiopulos, N., van de Vosse, F.N., Bovendeerd, P.H.M.: A method for the quantification of the pressure dependent 3D collagen configuration in the arterial adventitia. Journal of Structural Biology 180(2), 335-342 (2012)

41. Schriefl, A.J., Wolinski, H., Regitnig, P., Kohlwein, S.D., Holzapfel, G.A.: An automated approach for three-dimensional quantification of fibrillar structures in optically cleared soft biological tissues. Journal of The Royal Society Interface 10(80), 20120,760 (2013)

42. Schriefl, J., Reinisch, J., Sankaran, S., Pierce, D.M., Holzapfel, G.A.: Quantitative assessment of collagen fibre orientations from two-dimensional images of soft biological tissues. Journal of The Royal Society Interface 9, 3081-3093 (2012)

43. Stoller, P., Reiser, K.M., Celliers, P.M., Rubenchik, A.M.: Polarization-modulated second harmonic generation in collagen. Biophysical journal 82(6), 3330-3342 (2002)

44. Stylianopoulos, T., Barocas, V.H.: Multiscale, structure-based modeling for the elastic mechanical behavior of arterial walls. Journal of biomechanical engineering 129(4), 611$618(2007)$

45. Sutton, M., Ke, X., Lessner, S., Goldbach, M., Yost, M., Zhao, F., Schreier, H.: Strain field measurements on mouse carotid arteries using microscopic three-dimensional digital image correlation. Journal of Biomedical Materials Research Part A 84(1), 178-190 (2008)

46. Teng, Z., Tang, D., Zheng, J., Woodard, P.K., Hoffman, A.H.: An experimental study on the ultimate strength of the adventitia and media of human atherosclerotic carotid arteries in circumferential and axial directions. Journal of Biomechanics 42(15), 2535-2539 (2009)

47. Timmins, L.H., Wu, Q., Yeh, A.T., Moore, J.E., Greenwald, S.E.: Structural inhomogeneity and fiber orientation in the inner arterial media. American Journal of Physiology-Heart and Circulatory Physiology 298(5), H1537-H1545 (2010)

48. Tower, T.T., Neidert, M.R., Tranquillo, R.T.: Fiber alignment imaging during mechanical testing of soft tissues. Annals of Biomedical Engineering 30(10), 1221-1233 (2002)

49. Tsamis, A., Phillippi, J.A., Koch, R.G., Pasta, S., Amore, A.D., Watkins, S.C., Wagner, W.R., Gleason, T.G., Vorp, D.A.: Fiber micro-architecture in the longitudinal-radial and circumferential-radial planes of ascending thoracic aortic aneurysm media. Journal of Biomechanics 46(16), 2787-2794 (2013)

50. Van Zandvoort, M., Engels, W., Douma, K., Beckers, L., Oude Egbrink, M., Daemen, M., Slaaf, D.W.: Two-photon microscopy for imaging of the (atherosclerotic) vascular wall: A proof of concept study. Journal of Vascular Research 41(1), 54-63 (2004)

51. Venkatasubramanian, R.T., Grassl, E.D., Barocas, V.H., Lafontaine, D., Bischof, J.C.: Effects of freezing and cryopreservation on the mechanical properties of arteries. Annals of Biomedical engineering 34(5), 823-832 (2006) 
52. Voytik-Harbin, S.L., Roeder, B.A., Sturgis, J.E., Kokini, K., Robinson, J.P.: Simultaneous mechanical loading and confocal reflection microscopy for three-dimensional microbiomechanical analysis of biomaterials and tissue constructs. Microscopy and Microanalysis $\mathbf{9}$, $74-85(2003)$

53. Walker-Caprioglio, H., Trotter, J., Little, S., McGuffee, L.: Organization of cells and extracelluar matrix in mesenteric arteries of spontaneously hypertensive rats. Cell and Tissue Research 269(1), 141-149 (1992)

54. Wang, R., Brewster, L.P., Gleason, R.L.: In-situ characterization of the uncrimping process of arterial collagen fibers using two-photon confocal microscopy and digital image correlation. Journal of Biomechanics 46(15), 2726-2729 (2013)

55. Wasano, K., Yamamota, T.: Tridimensional architecture of elastic tissue in the rat aorta and femoral arterya scanning electron microscope study. Journal of Electron Microscopy 32(1), 33-44 (1983)

56. Wolinsky, H., Daly, M.M.: A method for the isolation of intima-media samples from arteries. Experimental Biology and Medicine 135(2), 364-368 (1970)

57. Zemánek, M., Burša, J., Děták, M.: Biaxial tension tests with soft tissues of arterial wall. Engineering Mechanics 16(1), 3-11 (2009)

58. Zoumi, A., Lu, X., Kassab, G.S., Tromberg, B.J.: Imaging coronary artery microstructure using second-harmonic and two-photon fluorescence microscopy. Biophysical Journal $\mathbf{8 7}(4), 2778-2786(2004)$ 